\title{
Comparing the Effects of Interactive and Noninteractive Complementary Nutrients on Growth in a Chemostat
}

\author{
James P. Braselton, Martha L. Abell, Lorraine M. Braselton \\ Department of Mathematical Sciences, Georgia Southern University, Statesboro, USA \\ Email: jbraselton@georgiasouthern.edu
}

Received July 12, 2013; revised August 12, 2012; accepted August 19, 2013

Copyright (C) 2013 James P. Braselton et al. This is an open access article distributed under the Creative Commons Attribution License, which permits unrestricted use, distribution, and reproduction in any medium, provided the original work is properly cited.

\begin{abstract}
We compare the effects of interactive and noninteractive complementary nutrients on the growth of an organism in the chemostat. We also compare these two situations to the case when the nutrients are substitutable. In previous studies, complementary nutrients have been assumed to be noninteractive. However, more recent research indicates that some complementary nutrient relationships are interactive. We show that interactive complementary and substitutable nutrients can lead to higher population densities than do noninteractive complementary nutrients. We numerically illustrate that if the washout rate is high, an organism can persist at higher densities when the complementary nutrients are interactive than when they are noninteractive, which can result in the extinction of the organism. Finally, we present an example by making a small adjustment to the model that leads to a single nutrient model with an intermediate metabolite of the original substrate as the nutrient for the organism.
\end{abstract}

Keywords: Chemostat; Growth; Dual Substrate; Complementary Nutrients; Substitutable Nutrients

\section{Introduction}

We consider a basic, resource-based model of growth in the chemostat. Such models have applications in ecology to model a simple lake and in biotechnology to model the commercial bio-reactor. Experimental verification of the match between theory and experiment in the chemostat can be found in [1]. Basic growth in the chemostat is described by the dimensionless system

$$
\begin{aligned}
& \frac{\mathrm{d} S}{\mathrm{~d} t}=\left(S^{(0)}-S\right) D-\frac{x}{y_{S}} f(S), S(0) \geq 0 \\
& \frac{\mathrm{d} x}{\mathrm{~d} t}=(f(S)-D) x, x(0) \geq 0 .
\end{aligned}
$$

For a detailed discussion of growth in the chemostat and a description of the constants $S^{(0)}$ (input of the nutrient), $y_{S}$ (yield constant), and $D$ (dilution (washout) rate), see Smith and Waltman [2].

Two nutrients are complementary if they meet different needs for an organism. For example, ammonia provides nitrogen while glucose provides carbon [3] (building blocks of protein). Similarly, two nutrients are substitutable if they meet the same needs for an organism. For example, glucose, galactose, maltose, ribose, arabinose, and fructose all provide energy (sugar) [4].

See Stroot et al. [5], for a recent study of Acinetobac- ter spp. bacteria in an activated sludge bioreactor system using the noninteractive Monod model for multiple nutrients.

However, other research [3,4,6-9] indicates that a model of interactive multiple limiting nutrients may be more appropriate for some situations. Of particular interest, Lendenmann and Egli [4] discuss several growth models appropriate for substitutable interactive nutrients and compare them to the growth of E. coli with sugar nutrients glucose, galactose, maltose, ribose, arabinose, and fructose. Whang et al. [9] perform a similar study using bacteria from the wastewater of a food-processing plant. On the other hand, Bapat et al. [10] use a Monod model to study the growth of $A$. mediterranei S699 with multiple interactive complementary nutrients. Champagne et al. [11] form a model of cometabolism with two interactive complementary nutrients in a well-mixed system. Bae and Rittmann [12] develop a dual-limiting model, compare the results to experimental data, and observe that they agree, which provides further evidence that a multiple nutrient limiting model might be more appropriate in some situations than a single nutrient limiting model. From [12], having an accurate kinetic model for dual limitation is essential for a proper design of treatment operations such as in bioremediation or contaminated groundwater... Dual limitation also can be 
critical for predicting the fate of pollutants in certain natural environments, such as a deep lake or an ocean...

To consider a single organism's growth in the chemostat for two nutrients, we study the dimensionless system

$$
\begin{aligned}
& \frac{\mathrm{d} S_{1}}{\mathrm{~d} t}=\left(S_{1}^{(0)}-S_{1}\right) D-\frac{x}{y_{1}} f\left(S_{1}, S_{2}\right), S_{1}(0) \geq 0 \\
& \frac{\mathrm{d} S_{2}}{\mathrm{~d} t}=\left(S_{2}{ }^{(0)}-S_{2}\right) D-\frac{x}{y_{2}} f\left(S_{1}, S_{2}\right), S_{1}(0) \geq 0 \\
& \frac{\mathrm{d} x}{\mathrm{~d} t}=\left(f\left(S_{1}, S_{2}\right)-D\right) x, x(0) \geq 0 .
\end{aligned}
$$

If the nutrients are complementary and noninteractive and assuming Monod (or Michaelis-Menten) kinetics typical choices of $f$ take the following forms. When assuming that the nutrients are noninteractive, one of the nutrients is the limiting nutrient. If the nutrients are complementary and noninteractive, we take $f$ to be

$$
f\left(S_{1}, S_{2}\right)=\min \left(\frac{m_{1} S_{1}}{K_{1}+S_{1}}, \frac{m_{2} S_{2}}{K_{2}+S_{2}}\right),
$$

The biological meaning of (3) is that one of $S_{1}$ or $S_{2}$ is the limiting nutrient, which is appropriate in modeling many situations [3]. If the nutrients are complementary and interactive, $f$ has the form

$$
f\left(S_{1}, S_{2}\right)=\frac{m_{1} S_{1}}{K_{1}+S_{1}} \frac{m_{2} S_{2}}{K_{2}+S_{2}} .
$$

Finally, if the nutrients are supplementary, $f$ has the form

$$
f\left(S_{1}, S_{2}\right)=\frac{m_{1} S_{1}}{K_{1}+S_{1}}+\frac{m_{2} S_{2}}{K_{2}+S_{2}} .
$$

and the constants are described in Table 1. (Refer to [13-
15] or [16]).

\section{Growth in the Chemostat}

Using Thieme's results from 1992 [17], we show that System (2) is asymptotic to a single nonlinear equation.

Let $\Sigma_{1}=S_{1}^{(0)}-S_{1}-\frac{1}{y_{1}} x$ and $\Sigma_{2}=S_{2}^{(0)}-S_{2}-\frac{1}{y_{2}} x$

Then, $\Sigma_{1}^{\prime}=-D \Sigma_{1}$ and $\Sigma_{2}^{\prime}=-D \Sigma_{2}$ so System (2) can be rewritten as

$$
\begin{aligned}
& \frac{\mathrm{d} \Sigma_{1}}{\mathrm{~d} t}=-D \Sigma_{1} \\
& \frac{\mathrm{d} \Sigma_{2}}{\mathrm{~d} t}=-D \Sigma_{2} \\
& \frac{\mathrm{d} x}{\mathrm{~d} t}=\left(f\left(S_{1}^{(0)}-\frac{1}{y_{1}} x-\Sigma_{1}, S_{2}^{(0)}-\frac{1}{y_{2}} x-\Sigma_{2}\right)-D\right) x
\end{aligned}
$$

Because the solutions of $\Sigma_{1}^{\prime}=-D \Sigma_{1}$ and $\Sigma_{2}^{\prime}=-D \Sigma_{2}$ are $\Sigma_{1}=C_{1} e^{-D t} \rightarrow 0$ as $t \rightarrow \infty$ and $\Sigma_{2}=C_{2} e^{-D t} \rightarrow 0$ as $t \rightarrow \infty$, in the limit as $t \rightarrow \infty$, System (6) is asymptotic to the equation

$$
\frac{\mathrm{d} x}{\mathrm{~d} t}=\left(f\left(S_{1}^{(0)}-\frac{1}{y_{1}} x, S_{2}^{(0)}-\frac{1}{y_{2}} x\right)-D\right) x
$$

Depending on whether the nutrients are complementary (noninteractive or interactive) or substitutable and assuming Monod (or Michaelis-Menten) kinetics typical choices of $f$ take the forms given by Equations (3)-(5). For all three situations, $x=0$ is a boundary rest point. If $f$ is given by (4), we the have the additional rest points: as the Equation (8)

Similarly, if $f$ is given by (5), we obtain the additional rest points: as the Equation (9)

$$
\begin{aligned}
x=\frac{1}{2\left(D-m_{1} m_{2}\right)}\left[D\left(K_{1}+S_{1}^{(0)}\right) y_{1}+D\left(K_{2}+S_{2}^{(0)}\right) y_{2}-m_{1} m_{2}\left(S_{1}^{(0)} y_{1}+S_{2}^{(0)} y_{2}\right)\right. & \\
& \pm\left(-4\left(D-m_{1} m_{2}\right)\left(-m_{1} m_{2} S_{1}^{(0)} S_{2}^{(0)}+D\left(K_{1}+S_{1}^{(0)}\right)\left(K_{2}+S_{2}^{(0)}\right)\right) y_{1} y_{2}\right. \\
& \left.\left.\quad+\left(m_{1} m_{2}\left(S_{1}^{(0)} y_{1}+S_{2}^{(0)} y_{2}\right)-D\left(\left(K_{1}+S_{1}^{(0)}\right) y_{1}+\left(K_{2}+S_{2}^{(0)}\right) y_{2}\right)\right)^{2}\right)^{1 / 2}\right] \\
x=\frac{1}{2}\left[K_{1} y_{1}+S_{1}^{(0)} y_{1}+K_{2} y_{2}+S_{2}^{(0)} y_{2}+\frac{K_{1} m_{1} y_{1}+K_{2} m_{2} y_{2}}{D-m_{1}-m_{2}}\right. & \\
& +\frac{1}{D-m_{1}-m_{2}}\left(-4\left(D-m_{1}-m_{2}\right)\left(-K_{2} m_{1} S_{1}^{(0)}-\left(K_{1} m_{2}+\left(m_{1}+m_{2}\right) S_{1}^{(0)}\right) S_{2}^{(0)}\right.\right. \\
& +D\left(K_{1}+S_{1}^{(0)}\right)\left(K_{2}+S_{2}^{(0)}\right) y_{1} y_{2}+\left(K_{1} m_{2} y_{1}+\left(m_{1}+m_{2}\right) S_{1}^{(0)} y_{1}\right. \\
& \left.\left.\left.\left.+K_{2} m_{1}+\left(m_{1}+m_{2}\right) S_{2}^{(0)} y_{2}-D\left(K_{1}+S_{1}^{(0)}\right) y_{1}+\left(K_{2}+S_{2}^{(0)}\right) y_{2}\right)\right)^{2}\right)^{1 / 2}\right]
\end{aligned}
$$


Numerical results using $f$ given by (3) (noninteractive complementary), (4) (interactive complementary), and (5) (substitutable) using the parameter values given in Table 2 are illustrated in Figure 1. In the figure, we can observe that substitutable nutrients lead to higher population densities than do complementary nutrients, which is not surprising. However, the differences in the densities from noninteractive and interactive nutrients are more surprising. When the nutrients are interactive, Rows 1 and 2 show that there can be a second (unstable) equilibrium population density. The stable population density is attained more quickly when the nutrients are interacttive than when they are noninteractive. Finally, the stable population density is generally higher when the nutrients are interactive than when they are noninteractive.

In fact, the population densities can be quite large as illustrated in Figure 2. Consider the values listed in Row 2 of Table 2 (corresponding to Row 2 of Figure 1). In Figure 2, we increase $D$ (the washout rate) from $D=$ 0.25 to $D=0.75$ (row 1 ), $D=1.25$ (row 2), and $D=1.75$ (row 3). Observe that as $D$ increases, interactive complementary nutrients lead to higher population densities than do noninteractive complementary nutrients. In fact, when the washout rate is sufficiently high, extinction occurs when the nutrients are noninteractive complementary while stable persistence occurs when the nutri-
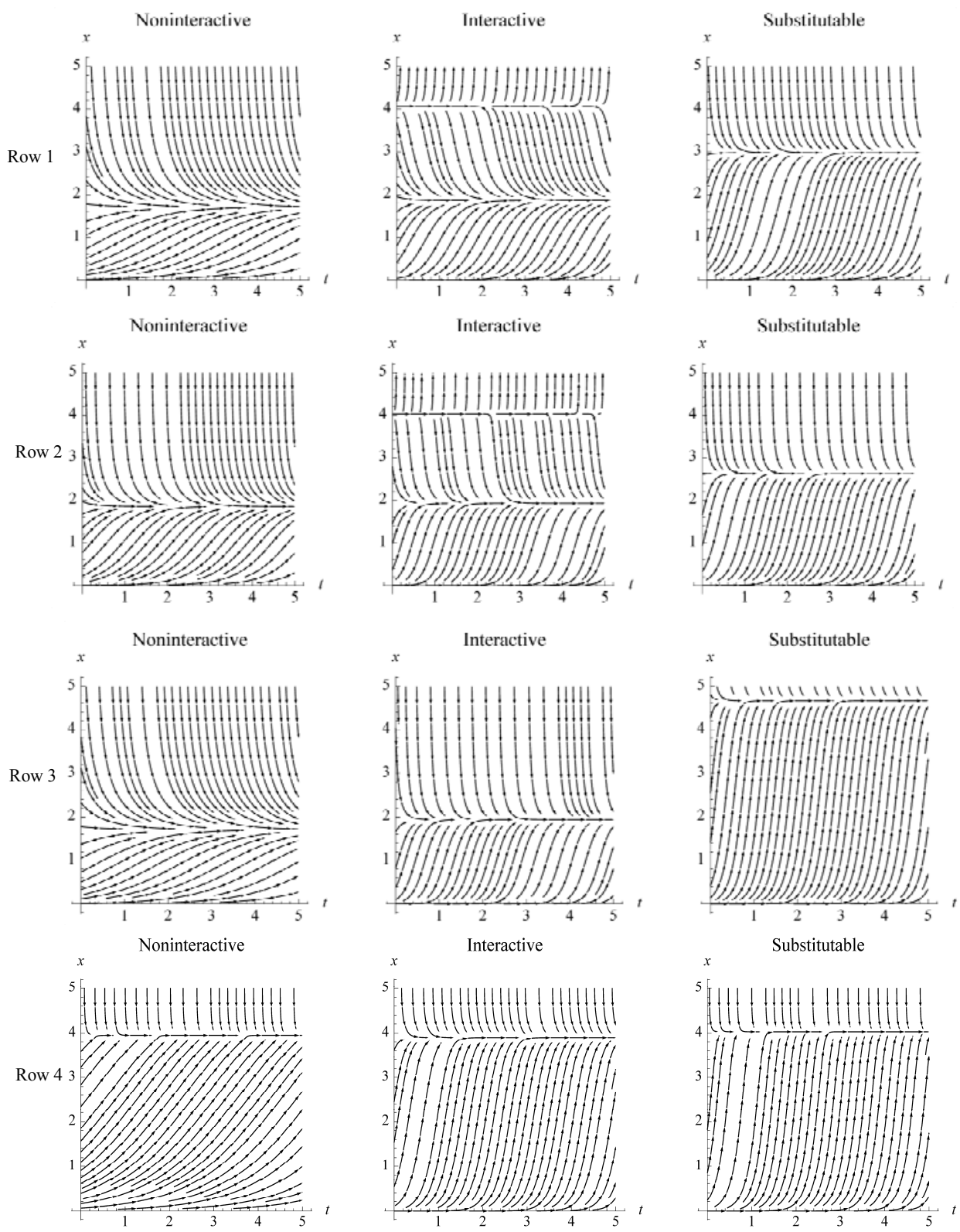

Figure 1. Modeling growth with noninteractive complementary, interactive complementary, and substitutable nutrients using the parameter values given in Table 2. 

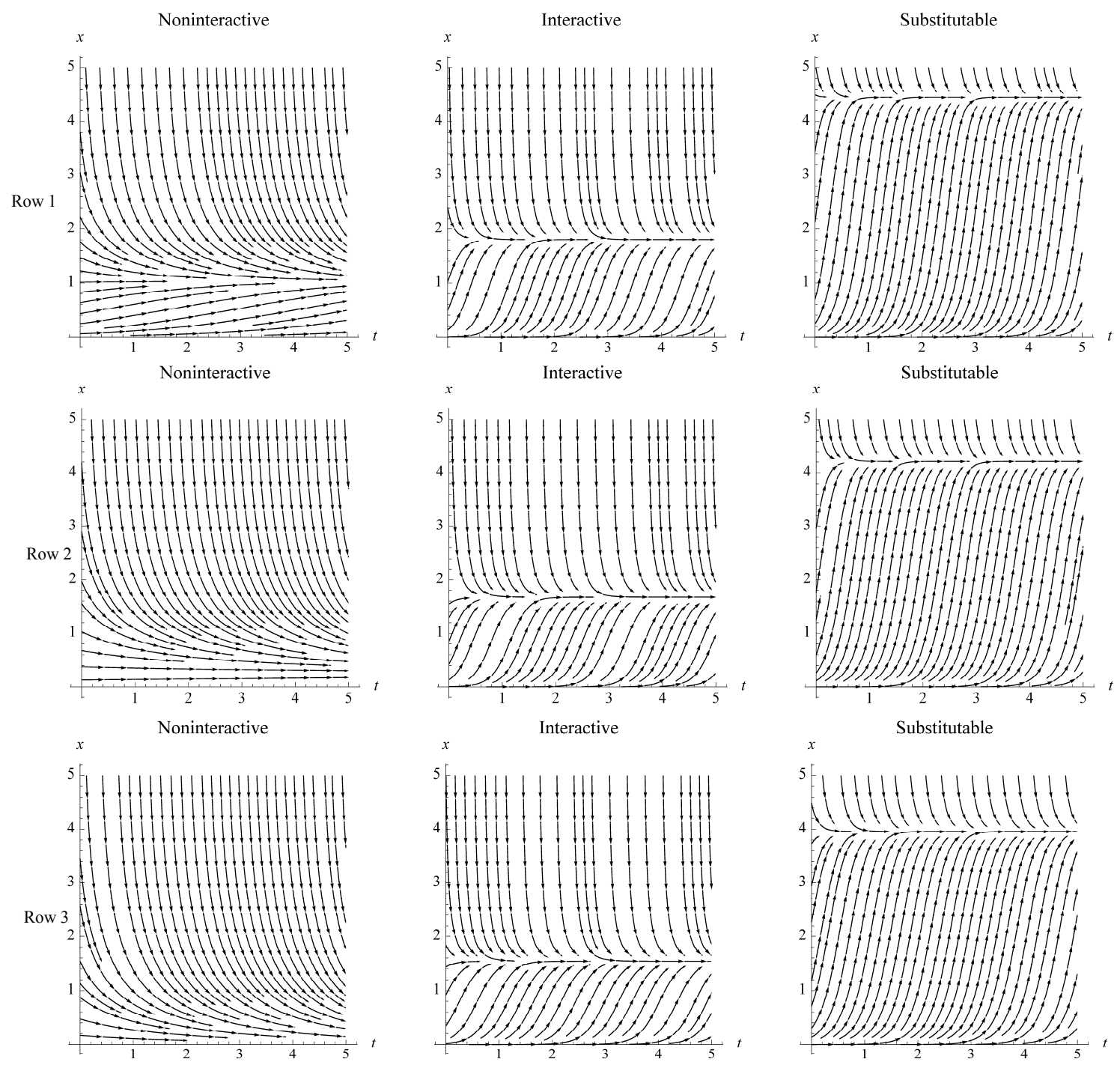

Figure 2. Row 1: $D=0.75$; Row 2: $D=1.25$; Row 3: $D=1.75$.

Table 1. Descriptions of the constants in Equations (2)-(5).

\begin{tabular}{cc}
\hline$S_{1}{ }^{(0)}, S_{2}{ }^{(0)}$ & Input concentration of the nutrients $S_{1}$ and $S_{2}$ \\
\hline$D$ & Dilution (washout) rate of chemostat \\
$m_{1}, m_{2}$ & Maximal growth rate of $i$ th competitor \\
$K_{1}, K_{2}$ & Michaelis-Menten (half-saturation) constants \\
$y_{1}, y_{2}$ & Yield constants \\
$f\left(S_{1}, S_{2}\right)$ & Growth rate \\
\hline
\end{tabular}

Entes are interactive and complementary.

\section{An Intermediate Metabolite}

A particularly interesting situation occurs when one substance degrades to a nutrient for the growth of an organism. Specifically, Sanchez et al. [18], study the particular situation in which phenol degrades to an intermediate metabolite that is then the primary nutrient for the organism (bacterium Pseudomonas putida Q5). This situation is particularly interesting because a "harmful" substance degrades to a state in which it is a nutrient for the organism under consideration that is growing in the chemostat, rendered harmless, and eliminated. To model this situation, System (2) is adjusted to

$$
\begin{aligned}
& \frac{\mathrm{d} S_{1}}{\mathrm{~d} t}=\left(S_{1}^{(0)}-S_{1}\right) D-\frac{x}{y_{1}} f_{1}\left(S_{1}, S_{2}\right), S_{1}(0) \geq 0 \\
& \frac{\mathrm{d} S_{2}}{\mathrm{~d} t}=-D S_{2}-\frac{x}{y_{1}} f_{1}\left(S_{1}, S_{2}\right)-f_{2}\left(S_{2}\right) x, S_{2}(0) \geq 0 \\
& \frac{\mathrm{d} x}{\mathrm{~d} t}=\left(f_{2}\left(S_{2}\right)-D\right) x, x(0) \geq 0,
\end{aligned}
$$

where $a>0$ is a positive constant. Sanchez et al. [18], successfully fit a model of the form of System (10) using 


$$
f_{1}\left(S_{1}, S_{2}\right)=\frac{m_{1} S_{1}}{K_{1 a}+S_{1}^{p}} \frac{S_{2}}{K_{1 b}+S_{1}^{n}}
$$

where

$$
p=p_{1}+\left(1+e^{-\left(S_{2}+p_{2}\right)}\right)^{-1}
$$

and

$$
n=n_{1}+\left(1+e^{-\left(S_{2}+n_{2}\right)}\right)^{-1}
$$

and $f_{2}=a \cdot S_{2}$ to data obtained from their study of bacterium Pseudomonas putida Q5. With $p=n=1$ and $m_{1}=$ $m_{1} m_{2}$ (Notation in Equations (3)-(5).) and $f_{2}=a \cdot S_{2}$, (11) is the same as (4).

Although we cannot reduce System (10) to a single equation as with System (2), we can reduce it to a system of two equations. To do so, let $\Sigma=S_{1}^{(0)}-S_{1}-S_{2}-x$. Then, $\Sigma^{\prime}=-D \Sigma$ and System (10) can rewritten as

$$
\begin{aligned}
& \frac{\mathrm{d} \Sigma}{\mathrm{d} t}=-D \Sigma \\
& \frac{\mathrm{d} S_{2}}{\mathrm{~d} t}=-D S_{2}-\frac{x}{y_{1}} f_{1}\left(S_{1}^{(0)}-S_{2}-x-\Sigma, S_{2}\right)-f_{2}\left(S_{2}\right) x \\
& \frac{\mathrm{d} x}{\mathrm{~d} t}=\left(f_{2}\left(S_{2}\right)-D\right) x
\end{aligned}
$$

Because the solution of $\Sigma^{\prime}=-D \Sigma$ is $\Sigma=C e^{-D t} \rightarrow 0$ as $t \rightarrow \infty$, in the limit as $t \rightarrow \infty$, System (12) is asymptotic to the system

$$
\begin{aligned}
& \frac{\mathrm{d} S_{2}}{\mathrm{~d} t}=-D S_{2}-\frac{x}{y_{1}} f_{1}\left(S_{1}^{(0)}-S_{2}-x, S_{2}\right)-f_{2}\left(S_{2}\right) x, \\
& \frac{\mathrm{d} x}{\mathrm{~d} t}=\left(f_{2}\left(S_{2}\right)-D\right) x .
\end{aligned}
$$

For the problem to be biologically meaningful, the feasible region is

$$
\Omega=\left\{\left(x, S_{2}\right) \mid x \geq 0, S_{2} \geq 0, S_{1}^{(0)}-S_{2}-x \geq 0\right\} .
$$

Assuming Monod kinetics, we now assume that $f_{1}$ takes the form given by (11) and that $n=p=1$ and that $f_{2}\left(S_{2}\right)=m_{2} S_{2} /\left(K_{2}+m_{2} S_{2}\right)$.

The rest points of System (13) are $E_{0_{2}}=(0,0)$ and potentially two interior rest points of the form

$$
E_{I}=\left(x^{*}, D K_{2} /\left(m_{2}-D\right)\right)
$$

where

Evaluated at $E_{0_{2}}$, the Jacobian has eigenvalues $\lambda_{1,2}=$ $-D$, so $E_{0_{2}}$ is always stable.

If we eliminated $S_{2}$ rather than $S_{1}$, the limiting system is

$$
\begin{aligned}
& \frac{\mathrm{d} S_{1}}{\mathrm{~d} t}=\left(S_{1}^{(0)}-S_{1}\right) D-\frac{x}{y_{1}} f_{1}\left(S_{1}, S_{1}^{(0)}-S-x\right) \\
& \frac{\mathrm{d} x}{\mathrm{~d} t}=\left(f_{2}\left(S_{1}^{(0)}-S_{1}-x\right)-D\right) x
\end{aligned}
$$

with feasible region

$$
\Omega=\left\{\left(x, S_{1}\right) \mid x \geq 0, S_{1} \geq 0, S_{1}^{(0)}-S_{1}-x \geq 0\right\} .
$$

Again, assuming that $f_{1}$ takes the form given by (11) and that $n=p=1$ and that $f_{2}\left(S_{2}\right)=m_{2} S_{2} /\left(K_{2}+S_{2}\right)$, we find that System (15) has rest points $E_{0_{1}}=\left(0, S_{1}^{(0)}\right)$,

Table 2. Parameter values used for Figure 1.

\begin{tabular}{llllllllll}
\hline & $D$ & $S_{1}{ }^{(0)}$ & $y_{1}$ & $m_{1}$ & $K_{1}$ & $S_{2}{ }^{(0)}$ & $y_{2}$ & $m_{2}$ & $K_{2}$ \\
\hline 1. & 0.25 & 4 & 1 & 6 & 4 & 2 & 1 & 7 & 4 \\
2. & 0.25 & 4 & 1 & 6 & 4 & 2 & 1 & 7 & 8 \\
3. & 0.25 & 4 & 2 & 6 & 1 & 2 & 1 & 7 & 8 \\
4. & 0.25 & 4 & 1 & 6 & 1 & 2 & 3 & 7 & 8 \\
\hline
\end{tabular}

$$
\begin{aligned}
& x^{*}=\left(( D - m _ { 2 } ) \left(K_{2} m_{1}\left(-m_{2} S_{1}^{(0)}+D\left(K_{2}+S_{1}^{(0)}\right)\right)\right.\right. \\
& \left.+\left(D\left(K_{1 b}-K_{2}\right)-K_{1 b} m_{2}\right)\left(-m_{2}\left(K_{1 a}+S_{1}^{(0)}\right)+D\left(K_{1 a}+2 K_{2}+S_{1}^{(0)}\right)\right) y_{1}\right) \\
& \pm\left[\left(D-m_{2}\right)^{2} K_{2}^{2} m_{1}^{2}\left(m_{2} S_{1}^{(0)}-D\left(K_{2}+S_{1}^{(0)}\right)\right)^{2}\right. \\
& +2 K_{2} m_{1}\left(D-m_{2}\right)\left(D\left(-K_{1 b}+K_{2}\right)+K_{1 b} m_{2}\right)\left(m_{2} S_{1}^{(0)}-\left(K_{1 a}+S_{1}^{(0)}\right)\right. \\
& \left.+D\left(K_{1 a}\left(K_{2}-S_{1}^{(0)}\right)-S_{1}^{(0)}\left(K_{2}+S_{1}^{(0)}\right)\right)\right) y_{1} \\
& \left.\left.\left.+\left(D-m_{2}\right)^{2}\left(D\left(-K_{1 b}+K_{2}\right)+K_{1 b} m_{2}\right)^{2}\left(K_{1 a}+S_{1}^{(0)}\right)^{2} y_{1}^{2}\right)\right)\right) /\left(2\left(D-m_{2}\right)^{2}\left(K_{1 b}\left(D-m_{2}\right) y_{1}\right)\right. \\
& \left.\left.+K_{2}\left(m_{1}-D y_{1}\right)\right)\right]^{1 / 2}
\end{aligned}
$$


and potentially two interior rest points of the form $E_{I}=\left(x^{*}, S_{1}^{*}\right)$, where

and

Evaluated at $E_{0_{2}}$, the Jacobian has eigenvalues $\lambda_{1,2}=$ $-D$, so $E_{0_{2}}$ is always stable.

Finally, if we had eliminated x rather than $S_{1}$ or $S_{2}$, the limiting system is

$$
\begin{aligned}
& \frac{\mathrm{d} S_{1}}{\mathrm{~d} t}=\left(S_{1}^{(0)}-S_{1}\right) D-\frac{S_{1}^{(0)}-S_{1}-S_{2}}{y_{1}} f_{1}\left(S_{1}, S_{2}\right) \\
& \frac{\mathrm{d} S_{2}}{\mathrm{~d} t}=-D S_{2}+\frac{S_{1}^{(0)}-S_{1}-S_{2}}{y_{1}} f_{1}\left(S_{1}, S_{2}\right) \\
& -f_{2}\left(S_{2}\right)\left(S_{1}^{(0)}-S_{1}-S_{2}\right) \\
& x^{*}=\frac{1}{\left(2\left(D-m_{2}\right)\left(K_{1 b}\left(D-m_{2}\right) y_{1}+K_{2}\left(m_{1}-D y_{1}\right)\right)\right)} \\
& \times\left[K_{2} m_{1}\left(-m_{2} S_{1}^{(0)}+D\left(K_{2}+S_{1}^{(0)}\right)\right)+\left(D\left(K_{1 b}-K_{2}\right)-K_{1 b} m_{2}\right)\left(-m_{2}\left(K_{1 a}+S_{1}^{(0)}\right)+D\left(K_{1 a}+2 K_{2}+S_{1}^{(0)}\right)\right) y_{1}\right. \\
& \pm\left(K_{2}^{2} m_{1}^{2}\left(m_{2} S_{1}^{(0)}-D\left(K_{2}+S_{1}^{(0)}\right)\right)^{2}\right. \\
& +2 K_{2} m_{1}\left(D-m_{2}\right)\left(D\left(-K_{1 b}+K_{2}\right)+K_{1 b} m_{2}\right)\left(m_{2} S_{1}^{(0)}\left(K_{1 a}+S_{1}^{(0)}\right)\right. \\
& \left.+D\left(K_{1 a}\left(K_{2}-S_{1}^{(0)}\right)-S_{1}^{(0)}\left(K_{2}+S_{1}^{(0)}\right)\right)\right) y_{1} \\
& \left.\left.+\left(D-m_{2}\right)^{2}\left(D\left(-K_{1 b}+K_{2}\right)+K_{1 b} m_{2}\right) \times\left(K_{1 a}+S_{1}^{(0)}\right)^{2} y_{1}^{2}\right)^{1 / 2}\right] \\
& S_{1}^{*}=\frac{1}{\left(2\left(D-m_{2}\right)\left(K_{1 b}\left(D-m_{2}\right) y_{1}+K_{2}\left(m_{1}-D y_{1}\right)\right)\right)} \\
& \times\left[K_{2} m_{1}\left(-m_{2} S_{1}^{(0)}+D\left(K_{2}+S_{1}^{(0)}\right)\right)+\left(-D+m_{2}\right)\left(D\left(-K_{1 b}+K_{2}\right)+K_{1 b} m_{2}\right)\left(-K_{1 a}+S_{1}^{(0)}\right) y_{1}\right. \\
& \mp\left(K_{2}^{2} m_{1}^{2}\left(m_{2} S_{1}^{(0)}-D\left(K_{2}+S_{1}^{(0)}\right)\right)^{2}\right. \\
& +2 K_{2} m_{1}\left(D-m_{2}\right)\left(D\left(-K_{1 b}+K_{2}\right)+K_{1 b} m_{2}\right)\left(m_{2} S_{1}^{(0)}\left(K_{1 a}+S_{1}^{(0)}\right)+D\left(K_{1 a}\left(K_{2}-S_{1}^{(0)}\right)-S_{1}^{(0)}\left(K_{2}+S_{1}^{(0)}\right)\right)\right) y_{1} \\
& \left.\left.+\left(D-m_{2}\right)^{2}\left(D\left(-K_{1 b}+K_{2}\right)+K_{1 b} m_{2}\right)^{2} \times\left(K_{1 a}+S_{1}^{(0)}\right)^{2} y_{1}^{2}\right)\right] \\
& S_{1}^{*}=\frac{1}{\left(2\left(D-m_{2}\right)\left(K_{1 b}\left(D-m_{2}\right) y_{1}+K_{2}\left(m_{1}-D y_{1}\right)\right)\right)} \\
& \times\left[K_{2} m_{1}\left(-m_{2} S_{1}^{(0)}+D\left(K_{2}+S_{1}^{(0)}\right)\right)+\left(-D+m_{2}\right)\left(D\left(-K_{1 b}+K_{2}\right)+K_{1 b} m_{2}\right)\left(-K_{1 a}+S_{1}^{(0)}\right) y_{1}\right. \\
& \pm\left(K_{2}^{2} m_{1}^{2}\left(m_{2} S_{1}^{(0)}-D\left(K_{2}+S_{1}^{(0)}\right)\right)^{2}\right. \\
& +2 K_{2} m_{1}\left(D-m_{2}\right)\left(D\left(-K_{1 b}+K_{2}\right)+K_{1 b} m_{2}\right)\left(m_{2} S_{1}^{(0)}\left(K_{1 a}+S_{1}^{(0)}\right)+D\left(K_{1 a}\left(K_{2}-S_{1}^{(0)}\right)-S_{1}^{(0)}\left(K_{2}+S_{1}^{(0)}\right)\right)\right) y_{1} \\
& \left.\left.+\left(D-m_{2}\right)^{2}\left(D\left(-K_{1 b}+K_{2}\right)+K_{1 b} m_{2}\right)^{2} \times\left(K_{1 a}+S_{1}^{(0)}\right)^{2} y_{1}^{2}\right)^{1 / 2}\right]
\end{aligned}
$$



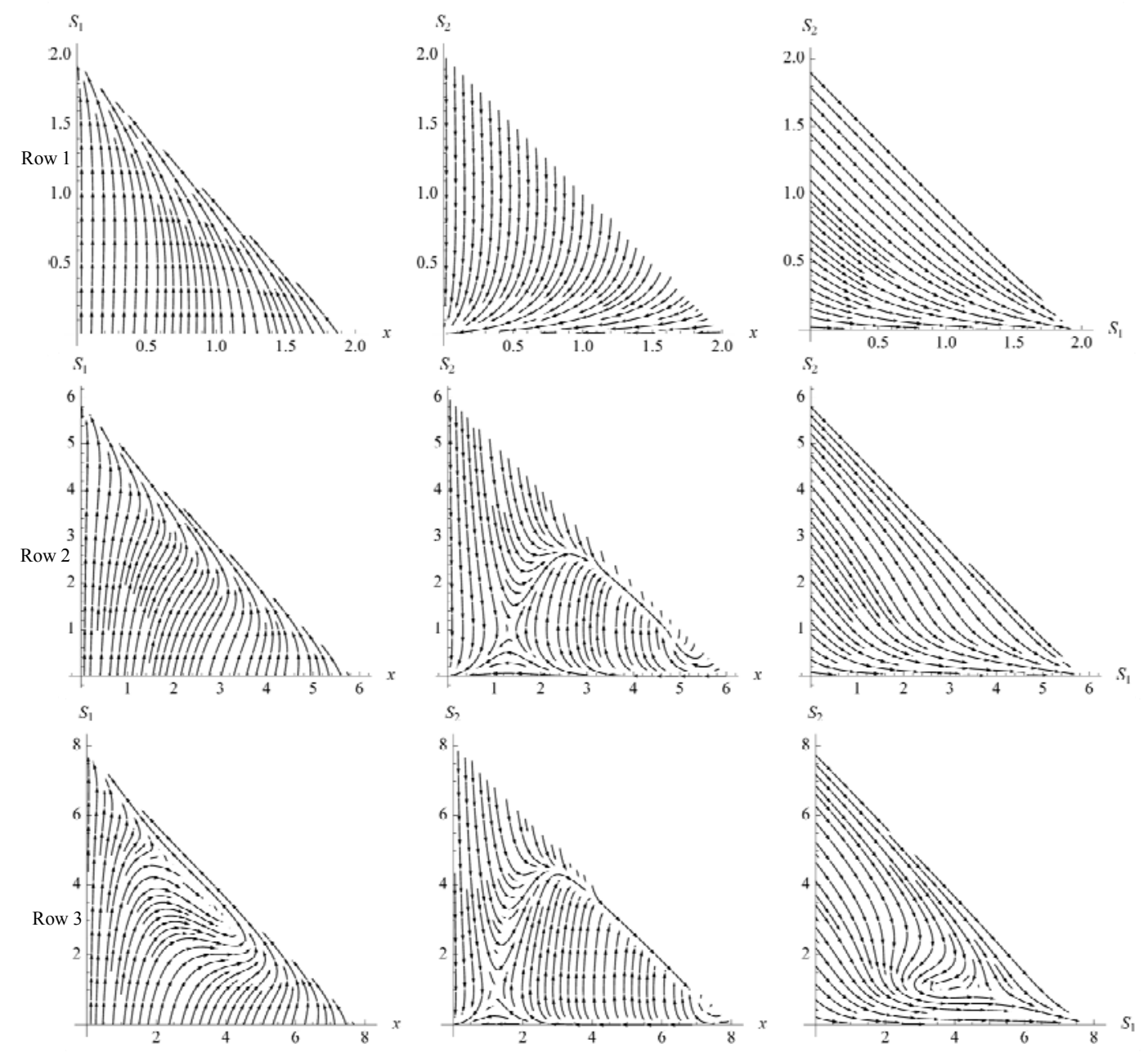

Figure 3. Modeling growth when the nutrient is an intermediate state of the substrate using the parameter values in Table 3.

Table 3. Parameter values used for Figure 3.

\begin{tabular}{lllllllll}
\hline & $D$ & $S_{1}{ }^{(0)}$ & $y_{1}$ & $m_{1}$ & $K_{1 a}$ & $K_{1 b}$ & $m_{2}$ & $K_{2}$ \\
\hline 1. & 0.5 & 2 & 1 & 3 & 2 & 2 & 1 & 1 \\
2. & 0.5 & 6 & 1 & 3 & 2 & 2 & 1 & 1 \\
3. & 0.5 & 8 & 1 & 3 & 2 & 2 & 1 & 1 \\
\hline
\end{tabular}

two cases there are no interior rest points. While in the third, when $S_{1}^{(0)}$ is increased to $8,(3,4),(3,1)$, and $(4,1)$, respectively, are degenerate rest points. For each, one eigenvalue of the Jacobian evaluated at the rest point is $\lambda_{1}$ $=-0.0833$ and the other is $\lambda_{2}=0$.

More interesting behavior of the systems is illustrated in Figure 4 where we use the parameter values in Table 4. The figure illustrates that when $S_{1}^{(0)}$ (input concentration of the substrate), $m_{1}$, or $m_{2}$ (growth rates) are increased, equilibrium states occur.

In all three situations, there is one unstable rest point, $\left(x, S_{1}, S_{2}\right)=(1.76,7.24,1),(1.54,9.46,1)$, and $(0.260$, $4.56,0.143)$ as shown in Table 5. However, in the first two rows of Table 5, $\left(x, S_{1}, S_{2}\right)=(6.23,2.76,1)$ and $(8.46,2.54,1)$ are stable spirals while in the third row, $(x$, $\left.S_{1}, S_{2}\right)=(2.92,1.93,0.143)$ is an unstable spiral.

Thus, depending on the parameter values equilibrium states may be stable or unstable. Moreover, slight adjustments to the parameter values might make a stable state unstable and vice-versa.

\section{Conclusions}

In this paper, we have formed a model of growth in a chemostat with two interactive complementary nutrients. We have used numerical results to graphically illustrate differences in the behavior of systems modeling noninteractive complementary nutrients, interactive complementary nutrients, and substitutable nutrients. Not surprisingly, we have illustrated that substitutable nutrients lead to higher population densities than do complementtary nutrients. We have illustrated some surprising differences in the behavior of systems modeling noninteractive complementary nutrients and interactive complementary nutrients. It is possible that if the nutrients are 

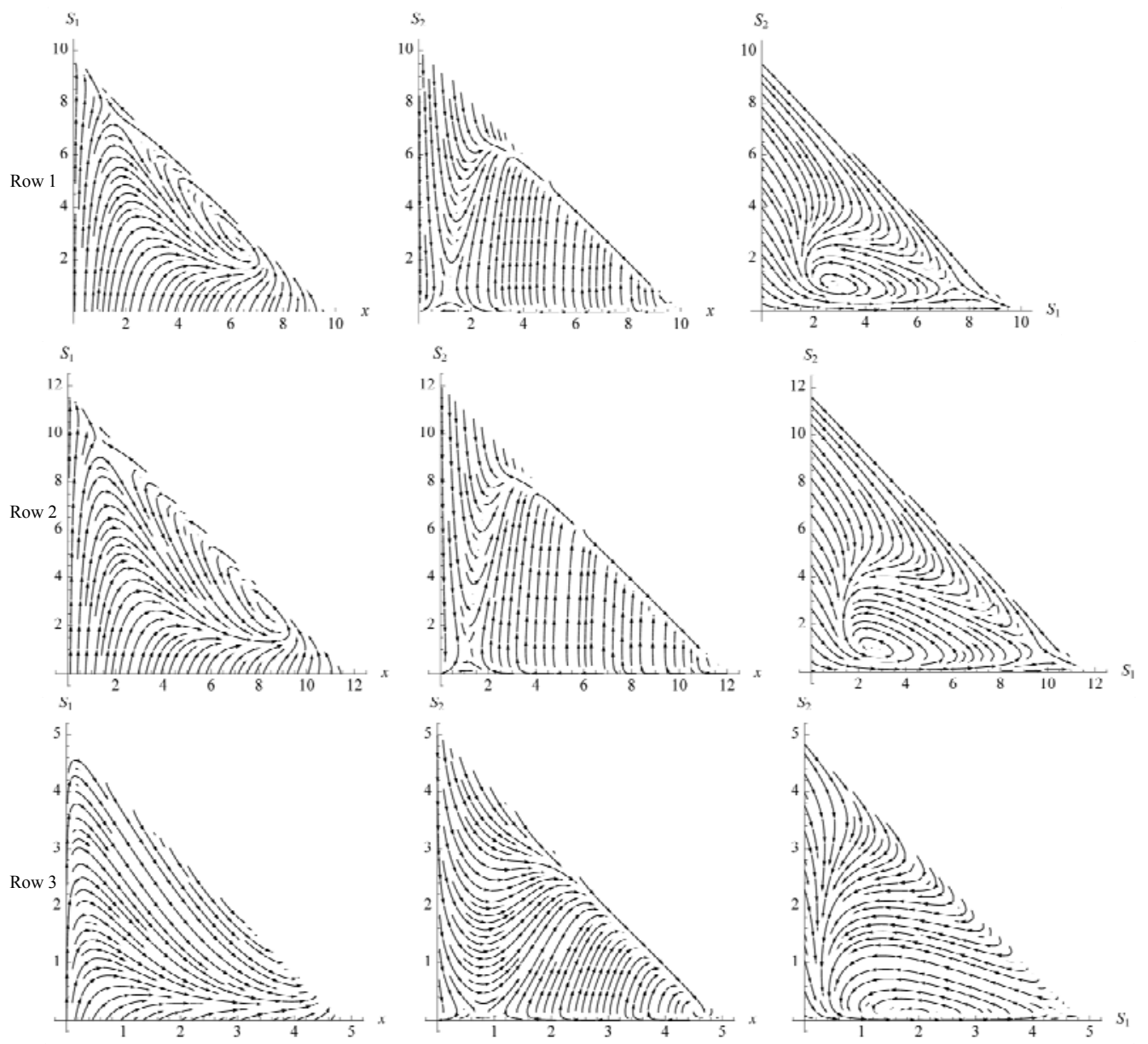

Figure 4. Modeling growth when the nutrient is an intermediate state of the substrate using the parameter values in Table 4.

Table 4. Parameter values used for Figure 4.

\begin{tabular}{lllllllll}
\hline & $D$ & $S_{1}{ }^{(0)}$ & $y_{1}$ & $m_{1}$ & $K_{1 a}$ & $K_{1 b}$ & $m_{2}$ & $K_{2}$ \\
\hline 1. & 0.5 & 10 & 1 & 3 & 2 & 2 & 1 & 1 \\
2. & 0.5 & 12 & 1 & 3 & 2 & 2 & 1 & 1 \\
3. & 0.25 & 5 & 1 & 8 & 2 & 2 & 2 & 1 \\
\hline
\end{tabular}

Table 5. Rest points and eigenvalues of Jacobian for Figure 4.

\begin{tabular}{ccccc}
\hline Row & $\mathrm{x}-S_{1}$ & $\mathrm{x}-S_{2}$ & $S_{1}-S_{2}$ & $\begin{array}{c}\text { Eigenvalues } \\
\text { of Jacobian }\end{array}$ \\
\hline \multirow{2}{*}{1} & $(1.76,7.24)$ & $(1.76,1)$ & $(7.24,1)$ & $-0.359,0.298$ \\
& $(6: 23,2: 76)$ & $(6.24,1)$ & $(2.76,1)$ & $\begin{array}{c}-0.983 \pm \\
0.850 i\end{array}$ \\
& & & & $-0.373,0.311$ \\
2 & $(1.54,9.46)$ & $(1.54,1)$ & $(9.46,1)$ & $-0.142 \pm$ \\
& $(8.46,2.54)$ & $(8.46,1)$ & $(2.54,1)$ & $1.26 i$ \\
& & & & \\
& $(0.260$, & $(0.296$, & $(4.56,1.43)$ & $0.230,-0.224$ \\
3 & $4.56)$ & $0.143)$ & & $0.0401 \pm$ \\
& $(2.92,1.93)$ & $(2.92$, & $(1.93,0.143)$ & $0.919 i$ \\
\hline
\end{tabular}

interactive, the organism will attain a stable population density while if the nutrients are noninteractive, the organism will become extinct. The simulations indicate that interactive complementary nutrients frequently lead to higher population densities than do noninteractive complementary nutrients. This agrees with the experimental results of Whang et al. [9] that show that complementary substrates (glucose and peptone) significantly increased hydrogen production by anaerobic hydrogenproducing bacteria.

A slight adjustment to the system leads to a completely different interpretation of the model in which the nutrient is an intermediate byproduct of the substrate. The simulations indicate the equilibrium states are very sensitive to the parameter values and slight adjustments to them can cause a stable state to become unstable and vice versa. This agrees with the experimental results of Sanchez et al. [18], which illustrated that slight adjustments of the inlet concentration (corresponding to $S_{1}^{(0)}$ ) had major effects on the washout rate (when the limit as $t \rightarrow \infty$ converges to $\left(x, S_{1}, S_{2}\right)=\left(0, S_{1}^{(0)}, 0\right)$.

The Mathematica notebooks that the authors used to carry out many of the calculations as well as generate the 
figures are available from the authors by sending an email request to Jim Braselton.

In future studies, we hope to examine how competitive relationships (competition, predator-prey, and so forth) are modeled under circumstances such as these.

\section{REFERENCES}

[1] S. R. Hansen and S. P. Hubbell, "Single Nutrient Microbial Competition: Agreement between Experimental and Theoretical Forecast Outcomes," Science, Vol. 20, No. 4438, 1980, pp. 1491-1493. http://dx.doi.org/10.1126/science.6767274

[2] H. L. Smith and P. Waltman, "The Theory of the Chemostat: Dynamics of Microbial Competition, Cambridge Studies in Mathematical Biology," Cambridge University Press, Cambridge, 1995. http://dx.doi.org/10.1017/CBO9780511530043

[3] C. P. L. Grady, Jr., G. T. Daigger and H. C. Lim, "Biological Wastewater Treatment," Second Edition, revised and expanded, Marcel Dekker, New York, 1999.

[4] U. Lendenmann and T. Egli, "Kinetic Models for the Growth of Escherichia Coli with Mixtures of Sugars Under Carbon-Limited Conditions," Biotechnology and Bioengineering, Vol. 59, No. 1, 1998, pp. 99-107. http://dx.doi.org/10.1002/(SICI)1097-0290(19980705)59: 1<99::AID-BIT13>3.0.CO;2-Y

[5] P. G. Stroot, P. E. Saikaly and D. B. Oerther, "Dynamic Growth Rates of Microbial Populations in Activated Sludge Systems," Journal of Environmental Engineering, Vol. 131, No. 12, 2005, pp. 1698-1705. http://dx.doi.org/10.1061/(ASCE)0733-9372(2005)131:12 (1698)

[6] W. Bae and B. E. Rittmann, "Effects of Electron Acceptor and Electron Donor on Biodegradation of CC14 by Biofilms," Environmental Engineering: Proceedings of the 1990 Specialty Conference, American Society of Civil Engineers, New York, 1990, pp. 390-395.

[7] J. L. Sherwood, J. N. Petersen, R. S. Skeen and N. B. Valentine, "Effects of Nitrate and Acetate Availability on Chloroform Production during Carbon Tetrachloride Destruction," Biotechnology and Bioengineering, Vol. 51, No. 5, 1996, pp. 551-557. http://dx.doi.org/10.1002/(SICI)1097-0290(19960905)51: 5<551::AID-BIT7>3.0.CO;2-B

[8] D. V. Vayenas and S. Pavlou, "Coexistence of Three Microbial Populations Competing for Three COMPLEMENTARY Nutrients in a Chemostat," Mathematical Biosciences, Vol. 161, No. 1-2, 1999, pp. 1-13. http://dx.doi.org/10.1016/S0025-5564(99)00040-1

[9] L.-M. Whang, C.-J. Hsiao and S.-S. Cheng, "A Dual-Substrate Steady-State Model for Biological Hydrogen Production in an Anaerobic Hydrogen Fermentation Proc- ess," Biotechnology and Bioengineering, Vol. 95, No. 3, 2006, pp. 492-500. http://dx.doi.org/10.1002/bit.21041

[10] P. M. Bapat, S. Bhartiya, K. V. Venkatesh and P. Wangikar, "Structured Kinetic Model to Represent the Utilization of Multiple Substrates in Complex Media During Rifamycin B Fermentation," Biotechnology and Bioengineering, Vol. 93, No. 4, 2006, pp. 779-790. http://dx.doi.org/10.1002/bit.20767

[11] P. Champagne, P. J. Van Geel and W. J. Parker, “A Proposed Transient Model for Cometabolism in Ciofilm Systems," Biotechnology and Bioengineering, Vol. 60, No. 5, 1998, pp. 541-550.

http://dx.doi.org/10.1002/(SICI)1097-0290(19981205)60: 5<541::AID-BIT4>3.0.CO;2-Q

[12] W. Bae and B. E. Rittmann, "A Structured Model of Dual-Limitation Kinetics," Biotechnology and Bioengineering, Vol. 49, No. 6, 1996, pp. 683-689.

http://dx.doi.org/10.1002/(SICI)1097-0290(19960320)49: 6<683::AID-BIT10>3.3.CO;2-E

[13] M. M. Ballyk and G. S. K. Wolkowicz, "Exploitive Competition in the Chemostat for Two Perfectly Substitutable Resources," Mathematical Biosciences, Vol. 118, No. 2, 1993, pp. 127-180. http://dx.doi.org/10.1016/0025-5564(93)90050-K

[14] G. J. Butler and G. S. K. Wolkowicz, "Exploitive Competition in a Chemostat for Two Complementary, and Possible Inhibitory, Resources," Mathematical Biosciences, Vol. 83, No. 1, 1987, pp. 1-48. http://dx.doi.org/10.1016/0025-5564(87)90002-2

[15] S-B. Hsu, K-S. Cheng and S. P. Hubbell, "Exploitive Competition for Two Complementary Nutrients in Continuous Cultures," SIAM Journal on Applied Mathematics, Vol. 41, No. 3, 1981, pp. 422-444. http://dx.doi.org/10.1137/0141036

[16] S.-B. Hsu and Y.-H. Tzeng, "Plasmid-Bearing, Plasmid-Free Organisms Competing for Two Complementary Nutrients in a Chemostat," Mathematical Biosciences, Vol. 179, No. 2, 2002, pp. 183-206. http://dx.doi.org/10.1016/S0025-5564(02)00105-0

[17] H. R. Thieme, "Convergence Results and a PoincaréBendixson Trichotomy for Asymptotically Autonomous Differential Equations," Journal of Mathematical Biology, Vol. 30, No. 7, 1992, pp. 755-763. http://dx.doi.org/10.1007/BF00173267

[18] J. L. Garcia-Sanchez, B. Kamp, K. A. Onysko, H. Budman and C. W. Robinson, "Double Inhibition Model for Degradation of Phenol by Pseudomonas Putida Q5," Biotechnology and Bioengineering, Vol. 60, No. 5, 1998, pp. 560-567.

http://dx.doi.org/10.1002/(SICI)1097-0290(19981205)60: 5<560::AID-BIT6>3.0.CO;2-L 